\title{
The infiltration pattern of microenvironmental cells and different immune escape mechanisms in colorectal cancer
}

1 Yihao Mao1, $\dagger$, Qingyang Feng1, 2, $\dagger$, Wenju Chang1, 2, $\dagger$, Yang Lv1, $\dagger$, Yuqiu Xu1, Jiang

2 Chang1, Peng Zheng1, Shanchao Yu1, Zhiyuan Zhang1, Zhiqiang Li3, Qi Lin1, 2, Wentao

3 Tang1, 2, Dexiang Zhu1, 2, Meiling Ji1, 2, Li Ren1, 2, Ye Wei1, 2, Guodong He 1, 2, * and

$4 \quad$ Jianmin Xu1, 2, *

$5{ }^{1}$ Department of General Surgery, Zhongshan Hospital, Fudan University, Shanghai, 200030, China

$6 \quad{ }^{2}$ Shanghai Engineering Research Center of Colorectal Cancer Minimally Invasive, Shanghai,

7 200030, China

$8{ }^{3}$ Department of General Surgery, No.2 Hospital, Nanping, 353000, China

$9+$ These authors contributed equally to this work.

10 * Correspondence:

11 Guodong He and Jianmin Xu

12 he.guodong@zs-hospital.sh.cn and xujmin@aliyun.com

13 Keywords: colorectal cancer, tumour microenvironment, immune escape, infiltration pattern, 14 immune subtypes.

15 Abstract

16 Background: The tumour microenvironment (TME) plays a crucial role in tumour progression and

17 metastasis. However, the infiltration pattern of TME cells in CRC samples and the immune escape

18 mechanism underneath have not been studied sufficiently.

19 Methods: Transcriptomic data from public datasets were retrieved online. In total, 1802 samples

20 from the microarray dataset and 619 samples from the TCGA dataset were enrolled. The ssGSEA

21 algorithm and unsupervised clustering were used for TME cells infiltration speculation and

22 infiltration pattern recognition. 
23 Results: CRC samples can be classified into three distinct TME cell subtypes. Subtype 1, the

24 immune-active subtype, was characterised by high infiltration of activated adaptive immune cells.

25 Subtype 2, the immune-desert subtype, featured high tumour purity and low infiltration of immune

26 and stromal cells. And subtype 3, stroma-rich subtype, had high infiltration of stromal cells. The

27 stroma-rich subtype conferred a significantly worse prognosis. Three subtypes had different immune

28 escape mechanisms. The immune-active subtype has the highest immune checkpoint expression

29 level. In comparison, the immune-desert subtype had the lowest immunogenicity and the defective

30 antigen presentation. And the stroma-rich subtype lacked activated immune cells.

31 Conclusions: Distinct TME cell subtypes and immune escape mechanisms may provide inspiration

32 and direction for further researches on CRC immunotherapy.

\section{$33 \quad 1$ Introduction}

34 Colorectal cancer (CRC) is estimated to be the third most commonly diagnosed and cause of death in

35 both men and women in the USA in 2021[1]. Tumour microenvironment (TME), defined as the

36 surrounding environment of a tumour, comprises tumour cells, immune cells, stromal cells, blood

37 vessels, and other mesenchymal cells. TME plays a crucial role in tumour progression and

38 metastasis[2].

39 Researchers have focused on CRC TME from multiple perspectives. Multiple TME cells were found

40 to have prognostic value and predict therapeutic benefit [3, 4]. As a classic example, Immunoscore,

41 which quantifies the in situ T cell infiltration in CRC tissue, was validated to be a valuable prognostic

42 factor[5] and was recommended in the European Society for Medical Oncology (ESMO)

43 guidelines[6]. However, numerous studies only focused on one or few types of TME cells. Moreover,

44 most of them were based on pathology level with a limited sample size. Confusing, even 
contradictory results were gotten. As CRC was a highly heterogeneous cancer, we assumed that the same type of TME cell might play different roles in different CRC tissues.

47 Recently, emerging papers explored the CRC TME using the bioinformatic based method, such as CIBERSORT[7], MCPcounter[8], TIMER[9], et al. However, because of the limitation of the above methods, like only relative infiltration results provided or limited predictable cell types, the landscape of TME cell infiltration pattern in CRC samples has not been systematically clarified to data, nor did

51 the immune escape mechanism underneath elucidated.

52 In this study, we comprehensively explored the TME cell infiltration pattern in two large independent

53 cohorts. CRC samples can be divided into three subtypes with distinct TME cell infiltration patterns

54 and their immune escape mechanism underneath. Also, the different clinical, prognostic, and 55 genomic characteristics were described.

\section{Materials and methods}

\section{$57 \quad 2.1 \quad$ Study population}

58 Public datasets selection criterion was described as follows: (1) transcriptomic data, including microarray data using Affymetrix HG-U133A (GEO accession number GPL96) or HG-U133 Plus

602.0 (GEO accession number GPL570) platforms or RNA-Seq data, were available; (2) the basic

61 clinicopathological information, including AJCC/UICC TNM stage and survival information (overall

62 survival (OS) or disease-free survival (DFS)) was available; (3) the sample size was larger than 50.

63 After systematically searching, GSE14333[10], GSE17538[11], GSE33113[12], GSE37892[13],

64 GSE38832[14], GSE39084[15], GSE39582[16], KFSYSCC, TCGA-COAD and TCGA-READ

65 datasets[17] were included in our research. Finally, 1802 samples from the microarray dataset and

66619 samples from the TCGA dataset were enrolled for subsequent analysis. The composition of each 
dataset was illustrated in Fig. S1. The microarray dataset was utilised to discover the infiltration

pattern of TME cells and perform survival-related analysis. Subsequent pattern validation and

mechanism exploration were conducted in the TCGA dataset. Due to the relatively shorter follow-up

time of the TCGA dataset (median, 21.9 months), survival-related analysis was only performed in the

71 microarray dataset.

\subsection{Data acquisition and preprocessing}

73 The transcriptome and clinical data of GSE14333, GSE17538, GSE33113, GSE37892, GSE38832,

GSE39084, and GSE39582 were downloaded from Gene Expression Omnibus (GEO) repository

(https://www.ncbi.nlm.nih.gov/gds/). The expression matrix and clinical information of KFSYSCC

were acquired from the supplementary material of a previous publication[18]. The RNA-Seq (RSEM

normalised), copy number variation (CNV), mutation, images of pathology slides, and clinical data

of TCGA-COAD and TCGA-READ datasets were downloaded from Genomic Data Commons

79 (GDC, https://portal.gdc.cancer.gov/) in Dec. 2019.

80 We used the voom function (in R package "limma" [19]) to transform RSEM normalised TCGA

81 RNA-Seq expression matrix. The ComBat algorithm ("ComBat" function in R package "sva" [20])

82 was applied to calibrate the heterogeneity among different datasets in microarray and TCGA datasets,

83 respectively. The PCA plots illustrating the effect of calibration were shown in Fig. S2.

\section{$84 \quad 2.3$ Microenvironmental cell infiltration speculation and validation}

85 We used the ssGSEA algorithm ("gsva" function in R package "GSVA" [21]) for infiltration

86 speculation of 31 microenvironmental cells based on transcriptomic data as previously described[22,

87 23]. Gene signature used in our study was mainly based on signature described in CHAROENTONG

88 P et al. [24], XIAO Y et al.[22] (which was a modification of LM22 signature of CIBERSORT) and

89 MCPcounter[25]. The detailed gene signature was described in Table S1. 
90 To validate the accuracy of our methods, we compared our calculation results with the estimation of

91 the cell abundances by CIBERSORT and MCPcounter (Table S2 and S3). The comparison results

92 showed high accordance between methods.late. These format

\section{$93 \quad 2.4$ Unsupervised clustering based on TME cell infiltration pattern}

94 For TME cell infiltration pattern discovery and validation, a nonnegative matrix factorisation

95 algorithm (NMF) ("nmf" function in R package "NMF" [26]) was performed for unsupervised

96 clustering on the scaled ssGSEA results. We used the "nmfEstimateRank" function (30 runs) to

97 choose the optimal number of clusters based on the Cophenetic correlation coefficient changing. The

98 highest clustering number before the Cophenetic correlation coefficient dropping most was selected

99 as the optimal clustering number.

\section{$100 \quad 2.5 \quad$ Slide Image analysis}

101 Hematoxylin and eosin (HE) stained diagnostic slides of the TCGA dataset were retrieved from GDC

102 Data Portal. The infiltration of tumour-infiltrating lymphocytes (TILs) was recorded as the mean

103 number of lymphocytes in tumour tissue from three random high power fields (HPF, 200×).

104 Furthermore, the infiltration of stromal cells was evaluated as the mean proportion of stromal cells in

105 tumour tissue from three randomised HPFs. Tumour necrosis in HE slides was characterised by

106 degraded tumour cells, presented as amorphous coagulum with nuclear debris (Fig 3G)[27].

107 Pathologists annotated the necrosis area in each diagnostic slide and the proportion of necrosis was

108 calculated by ImageJ (US National Institutes of Health, Bethesda, MD, USA). The above results

109 were all assessed by two independent pathologists who were blinded to the clinical data and the

110 results were averaged.

\section{$111 \quad 2.6 \quad$ Genomic analysis}


112 TCGA level 3 mutation data were downloaded from GDC Data Portal. The mutation load was

113 defined as $\log 2($ non-silent mutation number +1$)$. The neoantigen data was downloaded from The

114 Cancer Immunome Atlas (TCIA, https://tcia.at/)[28]. The cancer-testis antigen (CTA) scores,

115 homologous recombination deficiency (HRD) scores were downloaded from the supplementary

116 material of a previous publication[23]. The intratumor heterogeneity (ITH), defined as the subclonal

117 genome fraction measured by ABSOLUTE, was retrieved from an earlier publication[23]. The

118 GISTIC2.0 results of In silico Admixture Removal (ISAR) calibrated SCNV data (minus germline

$119 \mathrm{CNV}$ ) were obtained from a previous publication[23]. The GISTIC2.0 thresholded result of 2 or -2

120 was defined as deep $\mathrm{CN}$ alterations called amplifications and depletions. While the result of 1 or -1

121 was defined as shallow CN alterations, which were called gains and losses, respectively. The SCNV

122 load was calculated as the percentage of altered cytoband in each sample. T cell \& B cell receptor

123 (TCR\&BCR) diversity scores (Shannon Entropy, Evenness, and Richness) were obtained from a

124 previous publication[23]. Immune cytolytic activity score (CYT), which highly correlated with CD8+

125 T cell activation, was defined as the log-average (geometric mean) of GZMA and PRF1 expression

126 as previously described[29].

\section{$127 \quad 2.7$ Mutations and CNVs comparison between subtypes}

128 Mutations and CNVs were compared between every two subtypes. To adjust the difference of

129 mutation burden between subtypes, we adopted the method based on logistic regression as described

130 in a previous publication[22]. Specifically, the subtype was modelled as a logistic function as subtype

131 mutation burden + gene mutation status. If the P-value of a specific gene mutation status adjusted

132 for mutation burden less than 0.05 , such gene was defined as differentially mutated between

133 subtypes. Notably, gene mutations with an overall mutation rate of less than $2.5 \%$ were excluded

134 from the comparison. CNVs were compared similarly. Gains/amplifications and losses/deletions

135 were compared between every two subtypes separately. For gains/amplifications comparison, 
losses/deletions events were set to zero. The subtype was modelled as a logistic function as subtype

137 CNV burden + gene gain/amplification status. If the P-value of a specific gene gain/amplifications

138 adjusted for CNV burden less than 0.05, such gain/amplification was defined as differentially

139 distributed between subtypes. The losses/deletions comparisons were performed similarly. The above 140 calculations were performed using the "glm" function in R.

\section{$141 \quad 2.8 \quad$ Statistical analysis}

142 All statistical analyses were performed in R software, version 3.6.1 (The R Foundation for Statistical

143 Computing, http://www.rproject.org/). Continuous and ordered categorical variables were compared

144 using Student's t-tests or Kruskal-Wallis test with post hoc pairwise Dunn's-tests. Unordered

145 categorical variables were compared by Pearson $\chi^{2}$ test or Fisher exact test. Spearman correlation

146 analyses were employed for evaluating correlations between continuous variables. Survival analyses

147 were performed using Kaplan-Meier analyses and survival differences between groups were

148 compared by log-rank tests. Prognostic factors were identified by univariate and multivariate Cox

149 regression analyses. Factors with univariate regression $\mathrm{P}<0.1$ were further enrolled in multivariate

150 analyses. A two-sided $\mathrm{P}<0.05$ was considered statistically significant unless additionally stated.

\section{Results}

\section{$152 \quad 3.1$ Microenvironmental cell infiltration pattern in CRC}

153 We used the ssGSEA algorithm for estimating the absolute infiltration abundance of 31 different

154 TME cells in 1802 samples of the microarray dataset and 619 samples of the TCGA dataset. After

155 normalisation, a nonnegative matrix factorisation (NMF) algorithm, an unsupervised clustering

156 method, was applied to classify CRC samples into different subtypes based on TME cell infiltration.

157 The optimal cluster number of 3 was selected by the Cophenetic correlation coefficient in both

158 datasets (Figs. S3 and S4). Thus, we classified 1802 samples into three distinct TME cell (TMEC) 
subtypes (Fig. 1A). Subtype 1, named as immune-active subtype (marked by red), was characterised

160 by high infiltration of adaptive and innate immune cells, especially activated adaptive immune cells,

161 such as activated CD4 T cells, activated CD8 T cells and activated B cells, and low infiltration of

162 stromal cells. Subtype 2, named as immune-desert subtype (marked by blue), featured low infiltration

163 of most immune and stromal cells. And subtype 3, named as stroma-rich subtype (marked by

164 yellow), had high infiltration of adaptive and innate immune cells, as well as stromal cells but low

165 infiltration of activated adaptive immune cells compared with subtype 1 . The above infiltration

166 pattern was also validated in the TCGA dataset (Fig. 1B).

167 To further validate the above subtypes, we evaluated the pathological presentation of three distinct

168 subtypes in diagnostic slides of 499 TCGA samples. The representative pathology features of each

169 subtype were illustrated in Fig. 1C. Tumour tissue in subtype 1 featured median stromal proportion

170 with high tumor infiltrating leukocytes (TILs) infiltration. Subtype 2 had high tumour purity and low

171 TME cell infiltration. And mesenchymal cells infiltrated extensively in subtype 3 . Then we evaluated

172 the TILs and stromal cell infiltration pathologically in TCGA diagnostic slides. TILs infiltrated most

173 in subtype 1 and least in subtype 2 (median, 47 vs. 8 vs. 29, P<0.001), while subtype 3 had

174 significantly more stromal cell proportion (median, 0.047 vs. 0.037 vs. $0.098, \mathrm{P}<0.001$ ).

175 Further, we explored other characteristics of each subtype. We used the ESTIMATE algorithm to

176 calculate Immune Score, Stromal Score, and tumour purity for each sample. As shown in Fig. 1F,

177 Subtype 1 had the highest Immune Score, median Stromal Score, and relatively low tumour purity.

178 And subtype 2 featured the lowest Immune Score and Stromal Score but the highest tumour purity.

179 Subtype 3 had a median Immune Score, highest Stromal Score, and relatively low tumour purity (all

$180 \mathrm{P}<0.001)$. Also, The macrophage M1/M2 ratio was the highest in subtype $1(\mathrm{P}<0.001)$, while

181 Th17/Treg ratio was the highest in subtype $2(\mathrm{P}<0.001)$. Samples in subtype 3 own the highest 
182 Th1/Th2 ratio $(\mathrm{P}<0.001)$ and the proportion of activated adaptive immune cells was the highest in

183 subtype $1(\mathrm{P}<0.001)$. The above characteristics were all validated in the TCGA dataset (Fig. S6).

\subsection{TMEC Subtypes and clinicopathological features}

185 The correlation between TMEC subtypes and clinicopathological features of microarray and TCGA

186 datasets was presented in Table S4 and S5, respectively. Patients in subtype 1 were relatively older

187 (microarray dataset: mean $\pm \mathrm{SD}, 66.83 \pm 14.48$ vs. $65.87 \pm 13.70$ vs. $64.22 \pm 12.99, \mathrm{P}=0.005$; TCGA

188 dataset: mean \pm SD, $68.37 \pm 13.51$ vs. $65.25 \pm 10.98$ vs. $65.31 \pm 12.63, \mathrm{P}=0.019)$. Subtype 3 had more

189 samples with advanced stages ( $\mathrm{P}<0.001$ in both datasets). Subtype 1 was associated with more right

190 colon cancer $(\mathrm{P}<0.001$ in both datasets). Also, the dMMR/MSI-H samples were significantly

191 enriched in subtype 1 ( $\mathrm{P}<0.001$ in both datasets). Moreover, we compared the distribution of

192 Consensus Molecular Subgroups (CMS)[30] and TMEC subtypes, illustrated as Sankey plots in Fig.

$1931 \mathrm{G}$ and Fig. S7. Subtype 1 was mainly composed of CMS1 (MSI immune), CMS2 (Canonical), and

194 CMS3 (Metabolic) samples. The majority of subtype 2 samples were from CMS2. And samples of

195 CMS2 and CMS4 (Mesenchymal) constituted most of subtype 3. The above features were presented

196 in both microarray and TCGA datasets.

\section{$197 \quad 3.3 \quad$ TMEC Subtypes and CRC prognosis}

198 TMEC subtypes also plays an important role in CRC prognosis. The analyses related to overall

199 survival (OS) were performed in 1281 samples from

200 GSE17538/GSE38832/GSE39084/GSE39582/KFSYSCC with OS data available, while the analyses

201 related to disease-free survival (DFS) were performed in 1107 samples from

202 GSE14333/GSE17538/GSE33113/GSE38832/GSE39084/GSE39582 with DFS data available.

203 Subtype 3 conferred significantly worse OS and DFS, while subtype 1 and subtype 2 had similar

204 prognoses (Fig. 2A and 2B). Next, we conducted univariate and multivariate Cox regression of 
clinicopathological factors and TMEC subtypes for OS and DFS (Table S6 and Fig. 2C,

respectively). TMEC SUBTYPE of subtype 3 was a significant prognostic indicator in univariate

analyses of both $\mathrm{OS}(\mathrm{P}<0.001, \mathrm{HR}=1.449,95 \% \mathrm{CI}=1.174-1.787)$ and $\mathrm{DFS}(\mathrm{P}=0.002, \mathrm{HR}=1.456$,

95\% CI $=1.153-1.839)$. Multivariate analyses showed subtype 2 and subtype 3 were independent

prognostic factors of DFS $(\mathrm{P}=0.020, \mathrm{HR}=1.769,95 \% \mathrm{CI}=1.096-2.855$ and $\mathrm{P}=0.013, \mathrm{HR}=1.722,95 \%$

$\mathrm{CI}=1.121-2.646$, respectively) (Fig. 2C) but not OS (Table S6).

211 Subgroup analyses of OS and DFS revealed that subtype 3 indicated impaired survival in most

212 scenarios (Fig. S8). Furthermore, we analysed the prognostic value of different TME cells in all

213 samples and each subtype in the microarray dataset for OS (Fig. 2D) and DFS (Fig. 2E).

214 Interestingly, the same cell type may play converse prognostic roles in different TMEC subtypes. In

215 subtype 2, the majority of immune and stromal cells presented as protective factors of survival, even

216 immunoinhibitory cells like MDSCs and M2 macrophages, which indicated heterogeneous immune

217 networks in different TMEC subtypes.

\section{Possible immune escape mechanism of distinct TMEC subtypes}

219 The distinct characteristics of TMEC subtypes made us wonder whether they had different immune

220 escape mechanisms. Previous studies summarised vital factors leading to tumour immune escape as

221 follows: (1) defective antigen presentation; (2) tolerance and immune deviation; (3) infiltration of

222 immune-suppressive cells; (4) immune-suppressive mediators secretion [31, 32]. As multi-omics data

223 was available for the TCGA dataset, we explored the possible immune escape mechanism of three

224 TMEC subtypes.

\section{3.4.1 Defective antigen presentation}

226 Defective antigen presentation was composed of at least two aspects: alteration of tumour

227 immunogenicity and down-regulation of antigen presentation pathway. For tumour immunogenicity 
evaluation, subtype 1 had the highest mutation burden (Fig. 3A) and neoantigen load (Fig. 3B) (both

$\mathrm{P}<0.001$ ), which could be easily speculated for the highest proportion of dMMR/MSI-H samples.

230 While subtype 3 featured the highest SCNV load (Fig. 3C). The CTA and HRD scores of subtype 3

231 were higher than subtype $1(\mathrm{P}=0.008$ and $\mathrm{P}<0.001$, respectively) but not subtype 2 (both $\mathrm{P}>0.05)$

232 (Fig. 3D, Fig. 3E, respectively). Also, subtype 3 had the highest ITH level (P < 0.001) (Fig 3F). The

233 necrosis level between subtypes was not significantly differentiated $(P=0.167)$ (Fig. 3G). Subtype 2

234 showed the lowest BCR\&TCR richness diversity (both $\mathrm{P}<0.01$ ) (Fig. 3H), which were positively

235 correlated with cytolytic activity (Fig. 3I). As for antigen presentation pathway-related gene

236 expression, subtype 1 had higher expression of most MHC-related genes, while the expression of

237 subtype 2 was the lowest (all P < 0.01) (Fig. 3J). Overall, three subtypes all had impaired antigen

238 presentation to some extent, but subtype 2 had the lowest immunogenicity and antigen presentation

239 gene expression level.

\subsubsection{Tolerance and immune deviation}

241 Tumours were also known to induce immune tolerance by upregulating immune inhibitors and

242 downregulating immune stimulators. The relative expression levels and mean $\log 2$ (copy number)

243 value of immune inhibitors and immune stimulators were illustrated in Fig. 3J. Subtype 1 had the

244 highest expression level of most immune inhibitors and part of immune stimulators, which indicated

245 pro-tumour and anti-tumour immunity activation. The activation level of most above genes was the

246 lowest in subtype 2. Subtype 3 highly expressed TNF/TNF-receptors. Some differentially expressed

247 genes, such as SLAMF7, LAG3, CTLA4, BTLA, TNFSF8, et al. in two modules might attribute to

248 SCNV. Correlation analyses found that immune cytolytic activity was positively associated with

249 most immune checkpoint expression (Fig 3I). Generally, subtype 1 had a high expression of both

250 immune stimulators and immune inhibitors, whose expression level was the lowest in subtype 2. 


\subsubsection{Infiltration of immune-suppressive cells}

252 Next, we compared the infiltration of each cell type between subtypes, illustration as volcano plots

253 (Fig.4A). Comparing to subtype 2 and subtype 3, subtype 1 had significantly more activated CD4 and

254 CD8 T cells infiltration. Subtype 2, as an immune-cold subtype, had the least adaptive and innate

255 immune cells infiltration among three subtypes. For subtype 3, it had enriched fibroblasts and

256 endothelial cells than the other two subtypes. Immune-suppressive cells, such as Tregs, MDSCs, M2

257 macrophages, were more abundant in subtype 1 and 3. Also, we used the gene signature from a high-

258 quality publication[33] for exhausted T cells infiltration speculation and compared the infiltration of

259 exhausted T cells in each subtype (Fig. 4B). Subtype 1 had a significantly higher exhausted T cell

260 proportion than the other two subtypes $(\mathrm{P}<0.001)$, as well as the ratio of exhausted $\mathrm{T}$ cells to CD8 T

261 cells $(\mathrm{P}<0.001)$.

\section{$262 \quad 3.4 .4$ Immune suppressive mediators secretion}

263 Furthermore, we investigated the fourth aspect of the immune escape mechanism. The relative

264 expression levels to the median value and mean $\log 2$ (copy number) value of chemokines and

265 receptors, interferons and receptors, interleukins and receptors, and other cytokines in three subtypes

266 were compared, and cytokines with $\mathrm{P}<0.01$ was illustrated in Fig. 4E. Most cytokines and receptors

267 expression were negatively correlated between subtype 1 and subtype 3 , and the expression level of

268 subtype 2 was the lowest on most occasions. Subtype 1 expressed higher pro-immune chemokines

269 like CXCL9, CXCL10, CXCL11, which could recruit effector T cells and NK cells[34]. The IFN- $\gamma$,

270 which robustly stimulated anti-tumour immunity, was expressed higher in subtype 1. Pro-immunity

271 IL-2 and IL-15 were accumulated in subtype 1. IL-34, CCL2, CCL-3, CXCL8, and some of their

272 receptor CCR5, CXCR1, CXCR2, which promoted homeostasis of myeloid cells[35], were expressed

273 extensively in subtype 3. Mesenchymal development-related PDGFs and PDGFRs[36] and cell

274 adhesion-related SELP, ICAM1, ITGB2 were enriched in subtype 3. However, IDO, which 
contributed to peripheral tolerance, was significantly highly expressed in subtype 1 ( $\mathrm{P}<0.001)$ (Fig.

4C), indicating the existence of inhibitory cytokines in the inflamed microenvironment. Notably,

subtype 2 expressed a significantly higher level of VEGFA. Moreover, STING, a crucial factor in the

cGAS-Sting pathway, which contributes to the initiation of innate immunity and recognition of the

tumour, is highly expressed in subtype $1(\mathrm{P}<0.001)$ (Fig. 4D), suggesting the impaired immunity

initiation in subtype 2 and 3.

\section{1}

\subsection{TMEC subtype-specific genomic alterations}

Other than the heterogeneous immune escape mechanism among the TMEC subtypes, we further

explored the subtype-specific genomic alterations. First, we explored the activation of each subtype

in a comprehensive cancer development-related pathways collection [37, 38]. As presented in Fig.

5A, TP53, NRF2, PI3K pathways were upregulated in subtype 1, while MYC, PI3K pathways were

subtype 3. As for other cancer development-related pathways, cell cycle, genomic repair, protein

expression, metabolism, and immunity pathways were generally upregulated in subtype 1 . The

289 immunity and stromal pathways, which were highly enriched in subtype 3, were relatively

290 suppressed in subtype 2.

291 Next, we compared the subtype-specific gene mutations. Due to the high percentage of MSI-H

292 patients in subtype 1, it is easy to speculate that most mutations were enriched in subtype 1.

293 However, most mutations in MSI-H patients were random and irregular. Therefore, we focused on

294 the comparison among MSS/MSI-L patients. As shown in Fig. 5B, after adjusting for mutation

295 burden, KRAS, ZFHX4, SCN1A, MXRA5, WBSCR17, WDR87, COL5A2, KMT2B, DSCAML1,

296 KCNT2, PCDHGA3, CECR2, AKAP3, and GNAS were highly mutated in subtype 1. And the 
mutation frequency of HECW1, MYO5A, and ADAM29 was higher in subtype 2, While TP53

mutated more in subtype 3 (all adjusted $\mathrm{P}<0.05$ ).

299 As the CNV load of subtype 1 was significantly lower than the other two subtypes, the proportion of

300 alteration in most cytoband was the lowest among subtypes (Fig 5C). However, after adjusting for

301 CNV burden, most cytobands in Chr12 were more frequently amplificated in subtype 1. Also,

302 18p11.31-18q12.2 amplification was enriched in subtype 1. Subtype 2 exhibited more frequent

303 17p11.2-17q24.3 gain/amplification. Moreover, chr18 loss/deletion was more frequent in subtype 2

304 and 3.

\section{Discussion}

306 The development of personalised cancer medicine has garnered the concern of CRC subtyping. The

307 CRC subtyping system developed from pathological staging, mutation-based subtyping to omic-

308 based subtyping[30]. However, the large-scale comprehensive presentation of CRC TME cell

309 infiltration pattern and subsequent subtyping research was rare and insufficient. This study

310 systematically showed the infiltration pattern of primary TME cells in two large independent cohorts,

311 the microarray dataset and the TCGA dataset for cross-validation. The CRC samples can be mainly

312 divided into three subtypes, immune-active, immune-desert, and stroma-rich. The major

313 characteristics of each subtype were concluded in Fig. 6. The immune-active subtype features high

314 activated adaptive immune cells infiltrate, more right colon, more dMMR/MSI-H, and more CMS1

315 samples. The immune-desert subtype was characterised by low infiltration of most TME cells, more

316 left colon, and more CMS2. And the stroma-rich subtype presents the distinctiveness of high

317 infiltration of both immune cells and stromal cells, advanced stages, poor prognosis, and more CMS4

318 samples. Similar TME subtypes can also be observed in ovarian cancer[39] and TNBC[22]. 
319 The potential immune escape mechanism of each subtype may have important clinical implications.

320 The immune-active subtype has increased immune-suppressive cells, high immune-suppressive

321 cytokine levels, and upregulating immune inhibitors, implying both intense tumour-suppressing and

322 tumour-promoting immune responses existing. Theoretically, such subtype may benefit most from

323 immune checkpoint inhibitors (ICI), the most studied immunotherapy targeting TME in CRC, and

324 MSI status is a solid predictive marker for ICI efficacy[40]. However, not all dMMR/MSI-H patients

325 respond to anti-PD1/anti-PD-L1 therapy. Previous researches reported an ORR of $31 \%$ in refractory

$326 \mathrm{mCRC}[31]$ and 44\% in the first-line setting[41]. Most dMMR/MSI-H CRC is of immune-active

327 subtype, targeting the potential immune escape mechanism may be a novel strategy to improve the

328 efficacy. Strategies such as erasing the infiltration of immunosuppressive cells, neutralising

329 immunosuppressive cytokines, and combining other checkpoint inhibitors can be considered to

330 activate tumour immunity further.

331 The immune-desert subtype has decreased immune-promotive cells, low immune-promotive cytokine

332 level, downregulating immune inhibitors, and defective antigen presentation. Nevertheless, the OS is

333 similar to the immune-active subtype, implying the survival impairment of stromal cells has

334 neutralised the survival benefit of high infiltration of activated adaptive immune cells. Even the

335 infiltration of immunosuppressive cells, such as MDSC and Treg, may improve prognosis, which

336 shows the complexity and heterogeneity of CRC TME. The major difference between the immune-

337 desert subtype ("cold tumour") to other two subtypes ("hot tumour") is the antigen presentation

338 defect. Therefore, increasing tumour immunogenicity may induce the immune cell chemotaxis and

339 transform a "cold tumour" into a "hot tumour". Chemotherapy and radiotherapy can cause tumour

340 cell death with subsequent release of cellular fragmentation and cancer-associated neoantigens,

341 which are presented to APCs to increase tumour immunogenicity[42]. The use of pembrolizumab

342 plus FOLFOX achieved ORR 55\% in the first-line setting[43]. However, the synergistic effect of 
343 pembrolizumab and radiotherapy was not as expected, which only achieved an ORR of $4.5 \%$ in 22

344 pMMR/MSS mCRC patients[44]. Further researches of a dual checkpoint inhibitor (anti-PD-L1 +

345 anti-CTLA-4) following radiotherapy are underway (NCT02701400, NCT03122509). Other novel

346 approaches like tumour vaccine, and oncolytic virus are under early-phase research.

347 The stroma-rich subtype, which takes up nearly half the samples, has a similar immune escape

348 mechanism with the immune-active subtype but decreased immune-promotive cells. This subtype is

349 "hot" but owns the worst survival. The primary reason may be the pro-tumoural effect of excessive

350 stromal cell infiltration, excluding activated adaptive immune cells from the tumour. Anti-fibroblast,

351 anti-TGF- $\beta$ pathway, anti-angiogenesis, anti-immunosuppressive cytokines, or the combination of the

352 above therapy may be hoping to transform it into an immune-active subtype. Dual antagonising of

353 TGF- $\beta$ and PD-1/PD-L1 showed promising results in preclinical researches[45]. The combination of

354 vactosertib (a small-molecule inhibitor of TGF- $\beta$ ) and pembrolizumab showed an ORR of $15.2 \%$ in

355 previously treated MSS mCRC patients[46]. Furthermore, REGONIVO (regorafenib + nivolumab)

356 trial reported inspiring 36\% ORR in unselected mCRC patients[47], which implies the potential of

357 ICI and anti-angiogenesis. Previous studies showed that cancer-associated fibroblasts (CAFs) could

358 secret tumourigenic factors, modulate immunosuppressive TME, and create an ECM barrier to block

359 CD8 T cells accessing the tumour[48]. Multiple strategies targeting CAFs are under research, such as

360 normalising the phenotype of CAFs, inhibiting CAF generation and activation, reducing CAF

361 secretome[49], et al., and awaiting the results.

362 Also, the limitation of this study cannot be ignored. The major one is that the subtyping was based on

363 transcriptomic data, which needed to be further validated pathologically. Moreover, the immune

364 escape mechanism speculation was mainly based on in silico analysis, and numerous results only

365 reflected the correlation but not the causation. Further in vitro and in vivo experiments were needed. 
366 In conclusion, we systematically presented the infiltration pattern of CRC TME cells. CRC samples

367 can be divided into three subtypes with distinct TME cell infiltration patterns, clinical features,

368 genomic characteristics, as well as their immune escape mechanism underneath. The results may

369 provide inspiration and direction for further researches on CRC immunotherapy.

3705 Declarations

$371 \quad 5.1 \quad$ Ethics approval and consent to participate

372 Not applicable.

$373 \quad 5.2$ Consent for publication

374 All authors gave consent for publication.

\section{$375 \quad 5.3 \quad$ Availability of data and materials}

376 All datasets mentioned in this article were publicly available.

\section{$377 \quad 5.4 \quad$ Competing interests}

378 The authors declare that they have no competing interests.

\section{$379 \quad 5.5 \quad$ Funding}

380 This work was supported by the National Natural Science Foundation of China (No. 82002517,

381 82072678), Shanghai Sailing Program (20YF1407100), Clinical Research Plan of SHDC

382 (SHDC2020CR1033B, SHDC2020CR5006) and Shanghai Science and Technology Committee

383 Project (18140903200).

\section{$384 \quad 5.6 \quad$ Authors' contributions}


YM, GH and JX contributed to the planning of the study. YM, QF and YX collected the data and performed most of the bioinformatics analysis. YL and WC verified the numerical results by an independent implementation. YM, QF, YX, and YL drafted and revised the manuscript. All authors contributed to the interpretation of data and review of the manuscript. All authors reviewed and approved the final manuscript.

\subsection{Acknowledgements}

Not applicable.

393 1. Siegel RL, Miller KD, Fuchs HE, Jemal A: Cancer Statistics, 2021. CA: A Cancer Journal for Clinicians 2021, 71(1):7-33.

2. Quail DF, Joyce JA: Microenvironmental regulation of tumor progression and metastasis. Nat Med 2013, 19(11):1423-1437.

3. Feng Q, Chang W, Mao Y, He G, Zheng P, Tang W, Wei Y, Ren L, Zhu D, Ji M et al: Tumor-associated Macrophages as Prognostic and Predictive Biomarkers for Postoperative Adjuvant Chemotherapy in Patients with Stage II Colon Cancer. Clinical Cancer Research 2019, 25(13):3896-3907.

4. Bruni D, Angell HK, Galon J: The immune contexture and Immunoscore in cancer prognosis and therapeutic efficacy. Nat Rev Cancer 2020.

5. Pages F, Mlecnik B, Marliot F, Bindea G, Ou FS, Bifulco C, Lugli A, Zlobec I, Rau TT, Berger MD et al: International validation of the consensus Immunoscore for the classification of colon cancer: a prognostic and accuracy study. Lancet 2018, 391(10135):2128-2139.

6. Argiles G, Tabernero J, Labianca R, Hochhauser D, Salazar R, Iveson T, Laurent-Puig P, Quirke P, Yoshino T, Taieb J et al: Localised colon cancer: ESMO Clinical Practice Guidelines for diagnosis, treatment and follow-up. Ann Oncol 2020.

7. Newman AM, Liu CL, Green MR, Gentles AJ, Feng W, Xu Y, Hoang CD, Diehn M, Alizadeh AA: Robust enumeration of cell subsets from tissue expression profiles. Nat Methods 2015, 12(5):453-457.

8. Becht E, Giraldo NA, Lacroix L, Buttard B, Elarouci N, Petitprez F, Selves J, Laurent-Puig P, Sautes-Fridman C, Fridman WH et al: Estimating the population abundance of tissueinfiltrating immune and stromal cell populations using gene expression. Genome Biol 2016, 17(1):218.

9. Li T, Fan J, Wang B, Traugh N, Chen Q, Liu JS, Li B, Liu XS: TIMER: A Web Server for Comprehensive Analysis of Tumor-Infiltrating Immune Cells. Cancer Res 2017, 77(21):e108-e110. 
427

428

429

430

431

432

433

434

435

436

437

438

439

440

441

442

443

444

445

446

447

448

449

450

451

452

453

454

455

456

457

458

459

460

461

10. Jorissen RN, Gibbs P, Christie M, Prakash S, Lipton L, Desai J, Kerr D, Aaltonen LA, Arango D, Kruhøffer M et al: Metastasis-Associated Gene Expression Changes Predict Poor Outcomes in Patients with Dukes Stage B and C Colorectal Cancer. Clin Cancer Res 2009, 15(24):7642-7651.

11. Smith JJ, Deane NG, Wu F, Merchant NB, Zhang B, Jiang A, Lu P, Johnson JC, Schmidt C, Bailey CE et al: Experimentally derived metastasis gene expression profile predicts recurrence and death in patients with colon cancer. Gastroenterology 2010, 138(3):958968.

12. de Sousa E Melo F, Colak S, Buikhuisen J, Koster J, Cameron K, de Jong JH, Tuynman JB, Prasetyanti PR, Fessler E, van den Bergh SP et al: Methylation of cancer-stem-cellassociated Wnt target genes predicts poor prognosis in colorectal cancer patients. Cell Stem Cell 2011, 9(5):476-485.

13. Laibe S, Lagarde A, Ferrari A, Monges G, Birnbaum D, Olschwang S: A seven-gene signature aggregates a subgroup of stage II colon cancers with stage III. OMICS 2012, 16(10):560-565.

14. Tripathi MK, Deane NG, Zhu J, An H, Mima S, Wang X, Padmanabhan S, Shi Z, Prodduturi $\mathrm{N}$, Ciombor KK et al: Nuclear factor of activated T-cell activity is associated with metastatic capacity in colon cancer. Cancer Res 2014, 74(23):6947-6957.

15. Kirzin S, Marisa L, Guimbaud R, De Reynies A, Legrain M, Laurent-Puig P, Cordelier P, Pradère B, Bonnet D, Meggetto F et al: Sporadic early-onset colorectal cancer is a specific sub-type of cancer: a morphological, molecular and genetics study. PLOS ONE 2014, 9(8):e103159.

16. Marisa L, de Reyniès A, Duval A, Selves J, Gaub MP, Vescovo L, Etienne-Grimaldi M-C, Schiappa R, Guenot D, Ayadi M et al: Gene expression classification of colon cancer into molecular subtypes: characterization, validation, and prognostic value. PLoS Med 2013, 10(5):e1001453.

17. Cancer Genome Atlas N: Comprehensive molecular characterization of human colon and rectal cancer. Nature 2012, 487(7407):330-337.

18. Guinney J, Ferté C, Dry J, McEwen R, Manceau G, Kao KJ, Chang K-M, Bendtsen C, Hudson K, Huang E et al: Modeling RAS phenotype in colorectal cancer uncovers novel molecular traits of RAS dependency and improves prediction of response to targeted agents in patients. Clin Cancer Res 2014, 20(1):265-272.

19. Ritchie ME, Phipson B, Wu D, Hu Y, Law CW, Shi W, Smyth GK: limma powers differential expression analyses for RNA-sequencing and microarray studies. Nucleic Acids Res 2015, 43(7):e47.

20. Leek JT, Johnson WE, Parker HS, Jaffe AE, Storey JD: The sva package for removing batch effects and other unwanted variation in high-throughput experiments. Bioinformatics 2012, 28(6):882-883.

21. Hänzelmann S, Castelo R, Guinney J: GSVA: gene set variation analysis for microarray and RNA-seq data. BMC Bioinformatics 2013, 14:7.

22. Xiao Y, Ma D, Zhao S, Suo C, Shi J, Xue M-Z, Ruan M, Wang H, Zhao J, Li Q et al: MultiOmics Profiling Reveals Distinct Microenvironment Characterization and Suggests 
Immune Escape Mechanisms of Triple-Negative Breast Cancer. Clin Cancer Res 2019, 25(16):5002-5014.

24. Charoentong P, Finotello F, Angelova M, Mayer C, Efremova M, Rieder D, Hackl H, Immunophenotype Relationships and Predictors of Response to Checkpoint Blockade. Cell Rep 2017, 18(1):248-262.

Becht E, Giraldo NA, Lacroix L, Buttard B, Elarouci N, Petitprez F, Selves J, Laurent-Puig P, Sautès-Fridman C, Fridman WH et al: Estimating the population abundance of tissueinfiltrating immune and stromal cell populations using gene expression. Genome Biol 2016, 17(1):218.

27. Vayrynen SA, Vayrynen JP, Klintrup K, Makela J, Karttunen TJ, Tuomisto A, Makinen MJ: Gaujoux R, Seoighe C: A flexible R package for nonnegative matrix factorization. $B M C$ Bioinformatics 2010, 11:367. Clinical impact and network of determinants of tumour necrosis in colorectal cancer. $\mathrm{Br}$ J Cancer 2016, 114(12):1334-1342.

28. Charoentong P, Finotello F, Angelova M, Mayer C, Efremova M, Rieder D, Hack1 H, Trajanoski Z: Pan-cancer Immunogenomic Analyses Reveal GenotypeImmunophenotype Relationships and Predictors of Response to Checkpoint Blockade. Cell Rep 2017, 18(1):248-262.

29. Rooney MS, Shukla SA, Wu CJ, Getz G, Hacohen N: Molecular and genetic properties of tumors associated with local immune cytolytic activity. Cell 2015, 160(1-2):48-61.

30. Guinney J, Dienstmann R, Wang X, de Reynies A, Schlicker A, Soneson C, Marisa L, Roepman P, Nyamundanda G, Angelino P et al: The consensus molecular subtypes of colorectal cancer. Nat Med 2015, 21(11):1350-1356.

31. O'Donnell JS, Teng MWL, Smyth MJ: Cancer immunoediting and resistance to T cellbased immunotherapy. Nat Rev Clin Oncol 2019, 16(3):151-167.

32. Vinay DS, Ryan EP, Pawelec G, Talib WH, Stagg J, Elkord E, Lichtor T, Decker WK, Whelan RL, Kumara $\mathrm{H}$ et al: Immune evasion in cancer: Mechanistic basis and therapeutic strategies. Semin Cancer Biol 2015, 35 Suppl:S185-S198.

33. Newman AM, Steen CB, Liu CL, Gentles AJ, Chaudhuri AA, Scherer F, Khodadoust MS, Esfahani MS, Luca BA, Steiner D et al: Determining cell type abundance and expression from bulk tissues with digital cytometry. Nat Biotechnol 2019, 37(7):773-782.

34. Nagarsheth N, Wicha MS, Zou W: Chemokines in the cancer microenvironment and their relevance in cancer immunotherapy. Nat Rev Immunol 2017, 17(9):559-572.

35. Berraondo P, Sanmamed MF, Ochoa MC, Etxeberria I, Aznar MA, Pérez-Gracia JL, Rodríguez-Ruiz ME, Ponz-Sarvise M, Castañón E, Melero I: Cytokines in clinical cancer immunotherapy. Br J Cancer 2019, 120(1).

36. Manzat Saplacan RM, Balacescu L, Gherman C, Chira RI, Craiu A, Mircea PA, Lisencu C, Balacescu O: The Role of PDGFs and PDGFRs in Colorectal Cancer. Mediators Inflamm 2017, 2017:4708076. 
504

505

506

507

508

509

510

511

512

513

514

515

516

517

518

519

520

521

522

523

524

525

526

527

528

529

530

531

532

533

534

535

536

537

538

539

540

541

542

543

544

545

546

547

37. Sanchez-Vega F, Mina M, Armenia J, Chatila WK, Luna A, La KC, Dimitriadoy S, Liu DL, Kantheti HS, Saghafinia S et al: Oncogenic Signaling Pathways in The Cancer Genome Atlas. Cell 2018, 173(2):321-337 e310.

38. Tamborero D, Rubio-Perez C, Muiños F, Sabarinathan R, Piulats JM, Muntasell A, Dienstmann R, Lopez-Bigas N, Gonzalez-Perez A: A Pan-cancer Landscape of Interactions between Solid Tumors and Infiltrating Immune Cell Populations. Clin Cancer Res 2018, 24(15):3717-3728.

39. Desbois M, Udyavar AR, Ryner L, Kozlowski C, Guan Y, Dürrbaum M, Lu S, Fortin JP, Koeppen $\mathrm{H}$, Ziai $\mathrm{J}$ et al: Integrated digital pathology and transcriptome analysis identifies molecular mediators of T-cell exclusion in ovarian cancer. Nat Commun 2020, 11(1):5583.

40. Sahin IH, Akce M, Alese O, Shaib W, Lesinski GB, El-Rayes B, Wu C: Immune checkpoint inhibitors for the treatment of MSI-H/MMR-D colorectal cancer and a perspective on resistance mechanisms. Br J Cancer 2019, 121(10):809-818.

41. Shiu K-K, Andre T, Kim TW, Jensen BV, Jensen LH, Punt CJA, Smith DM, GarciaCarbonero R, Benavides M, Gibbs P et al: KEYNOTE-177: Phase III randomized study of pembrolizumab versus chemotherapy for microsatellite instability-high advanced colorectal cancer. Journal of Clinical Oncology 2021, 39(3_suppl):6-6.

42. Franke AJ, Skelton WP, Starr JS, Parekh H, Lee JJ, Overman MJ, Allegra C, George TJ: Immunotherapy for Colorectal Cancer: A Review of Current and Novel Therapeutic Approaches. J Natl Cancer Inst 2019, 111(11):1131-1141.

43. Shahda S, Noonan AM, Bekaii-Saab TS, O'Neil BH, Sehdev A, Shaib WL, Helft PR, Loehrer PJ, Tong Y, Liu Z et al: A phase II study of pembrolizumab in combination with mFOLFOX6 for patients with advanced colorectal cancer. Journal of Clinical Oncology 2017, 35(15_suppl):3541-3541.

44. Segal NH, Kemeny NE, Cercek A, Reidy DL, Raasch PJ, Warren P, Hrabovsky AE, Campbell N, Shia J, Goodman KA et al: Non-randomized phase II study to assess the efficacy of pembrolizumab (Pem) plus radiotherapy (RT) or ablation in mismatch repair proficient (pMMR) metastatic colorectal cancer (mCRC) patients. Journal of Clinical Oncology 2016, 34(15_suppl):3539-3539.

45. Liu S, Ren J, Ten Dijke P: Targeting TGF $\beta$ signal transduction for cancer therapy. Signal Transduct Target Ther 2021, 6(1):8.

46. Kim TW, Lee KW, Ahn JB, Lee J, Ryu J, Oh B, Ock C-Y, Hwang S, Hahm KB, Kim S-J et $a l$ : Efficacy and safety of vactosertib and pembrolizumab combination in patients with previously treated microsatellite stable metastatic colorectal cancer. Journal of Clinical Oncology 2021, 39(15_suppl):3573-3573.

47. Fukuoka S, Hara H, Takahashi N, Kojima T, Kawazoe A, Asayama M, Yoshii T, Kotani D, Tamura H, Mikamoto Y et al: Regorafenib Plus Nivolumab in Patients With Advanced Gastric or Colorectal Cancer: An Open-Label, Dose-Escalation, and Dose-Expansion Phase Ib Trial (REGONIVO, EPOC1603). J Clin Oncol 2020, 38(18):2053-2061.

48. Ford K, Hanley CJ, Mellone M, Szyndralewiez C, Heitz F, Wiesel P, Wood O, Machado M, Lopez M-A, Ganesan A-P et al: NOX4 Inhibition Potentiates Immunotherapy by Overcoming Cancer-Associated Fibroblast-Mediated CD8 T-cell Exclusion from Tumors. Cancer Res 2020, 80(9):1846-1860. 
49. Domen A, Quatannens D, Zanivan S, Deben C, Van Audenaerde J, Smits E, Wouters A, Lardon F, Roeyen G, Verhoeven Y et al: Cancer-Associated Fibroblasts as a Common Orchestrator of Therapy Resistance in Lung and Pancreatic Cancer. Cancers (Basel) 2021, 13(5).

\section{Captions}

554

555

556

557

558

559

560

561

562

563

564

565

566

567

568

569

570

571

572

573

574

575

576

577

578

579

580

581

582

583

584
Figure 1. The landscape of TME cell infiltration pattern in CRC. (A-B) Consensus heatmap illustrating the infiltration of 31 TME cells in the microarray dataset (A) and the TCGA dataset (B). Both of them were divided into three subtypes. (C) The representative pathology presentation of each subtype in the TCGA dataset (red, subtype 1; blue, subtype 2; yellow, subtype3; from top to bottom). (D) The number of TILs among three subtypes per high power field (HPF, 200×) in the TCGA dataset. (E) The proportion of stromal cells among three subtypes per high power field (HPF, 200×) in the TCGA dataset. (F) The distribution of Immune Score, Stromal Score, tumour purity, macrophage $\mathrm{M} 1 / \mathrm{M} 2$ ratio, Th17/Treg ratio, Th1/Th2 ratio, adaptive immune cells, activated adaptive immune cells, innate immune cells, and stromal cells among subtypes in the microarray dataset. $(\mathrm{G})$ Sankey plot illustrating the distribution of CMS subtypes and TMEC subtypes in the microarray dataset.

Figure 2. (A-B) Kaplan-Meier analysis of OS (A) and DFS (B) in the microarray dataset. (C) Univariate and multivariate Cox regression of clinicopathological factors and TMEC subtypes for DFS in the microarray dataset. (D-E) The prognostic value of different TME cells in all samples and each subtype in the microarray dataset for OS (D) and DFS (E).

Figure 3. (A-F) The mutation load (A), neoantigen load (B), SCNV load (C), CTA (D), HRD (E) and ITH (F) among TMEC subtypes. (G) The representative pathology image of tumor necrosis (left) and the distribution of necrosis percentage of each subtype. (H) The BCR and TCR richness of different subtypes. (I) The correlation between immune cytolytic activity and tumour immunogenicity factors, TILs, stromal cells, and immune checkpoint molecules. (J) The relative expression levels to the median value and mean $\log 2$ (copy number) value of immune inhibitors and immune stimulators among three subtypes. ***: $\mathrm{P}<0.001$; **: $0.001<\mathrm{P}<0.01$; *: $0.01<\mathrm{P}<0.05$; ns: $\mathrm{P}>0.05$.

Figure 4. (A) The comparison of each cell type between subtypes. (B) The distribution of exhausted $\mathrm{T}$ cells infiltration and the ratio of exhausted T cells to CD8 T cells infiltration among each subtype. (C-D) The expression of IDO and STING among each subtype. (E) The relative expression levels to the median value and mean $\log 2$ (copy number) value of chemokines, interleukins, other cytokines and their receptors among three subtypes. ***: $\mathrm{P}<0.001$; **: $0.001<\mathrm{P}<0.01 ; *: 0.01<\mathrm{P}<0.05$; ns: $\mathrm{P}>0.05$. 
586 Figure 5. TMEC subtype-specific genomic alterations. (A) Heatmap illustrating activation of

587 comprehensive cancer development-related pathways among three TMEC subtypes. (B) Subtype-

588 specific gene mutation frequency among MSS/MSI-L samples in three TMEC subtypes. (C)

589 Subtype-specific SCNVs of each TMEC subtype (top three plots) and comparison of SCNVs

590 between every two subtypes.

591

592 Figure 6. Summary of clinical features, immune escape mechanisms and subtype-specific genomic 593 alterations of three TMEC subtypes. 


\section{A Microarray dataset}

Subtype 1 Subtype 2 Subtype 3

Immune-active Immune-desert Stroma-rich

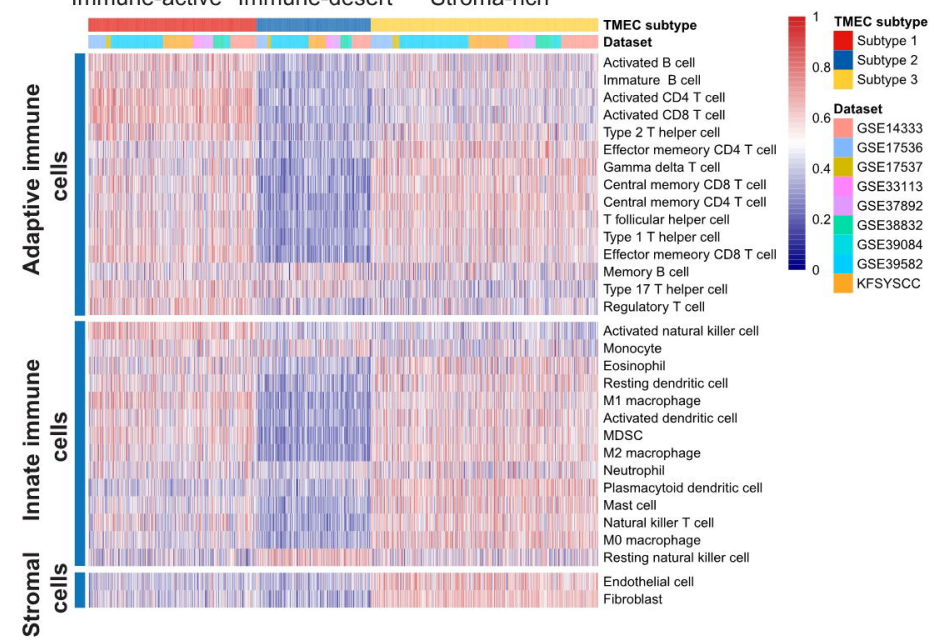

B

\section{TCGA dataset}

Subtype 1 Subtype 2 Subtype 3

Immune-active Immune-desert Stroma-rich

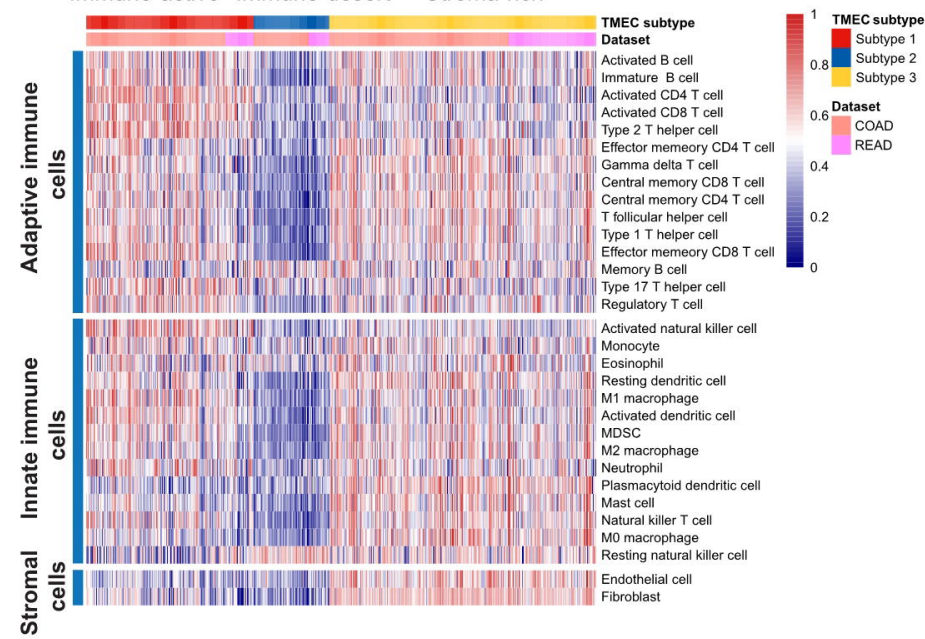

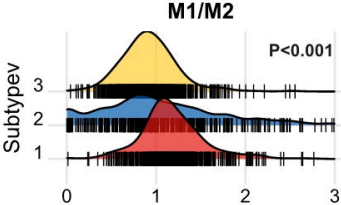

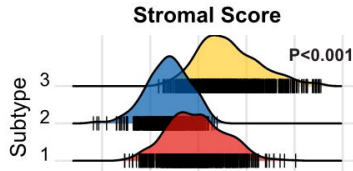

$-2000-1000 \quad 0 \quad 100020003000$
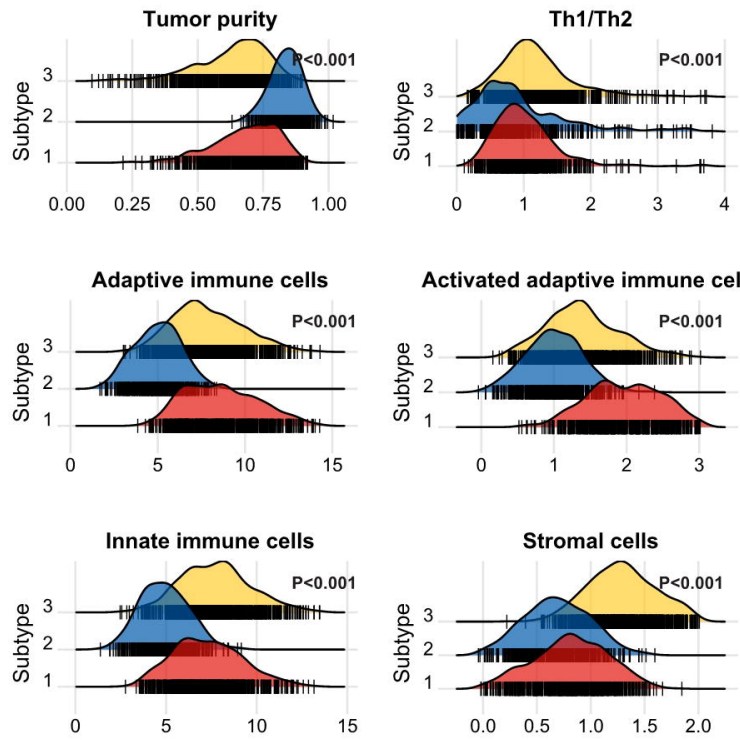

C

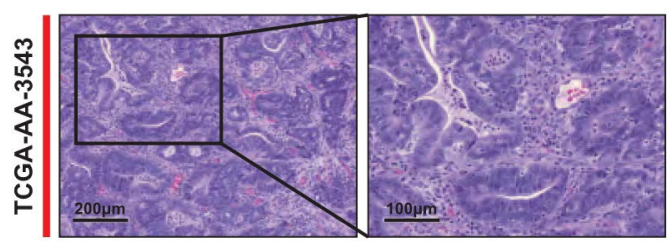

눙

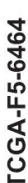
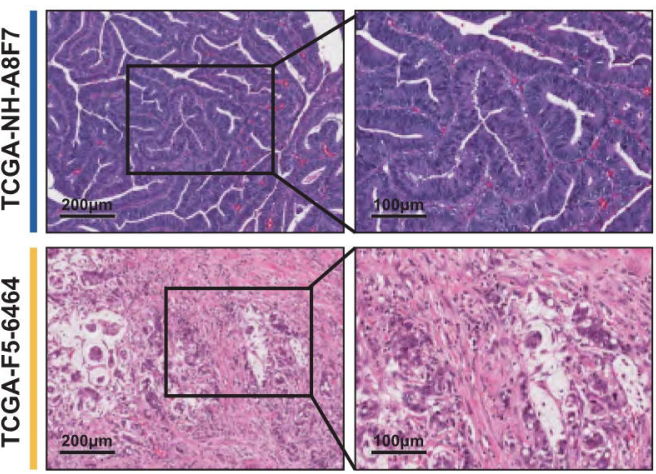

D

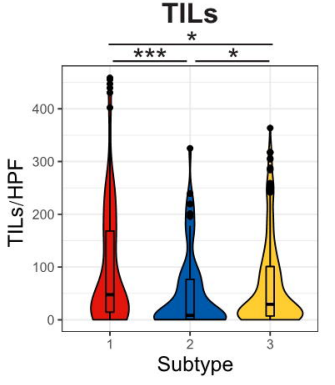

E

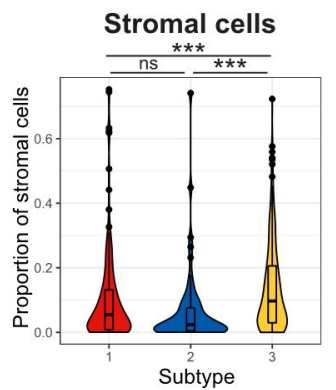

G

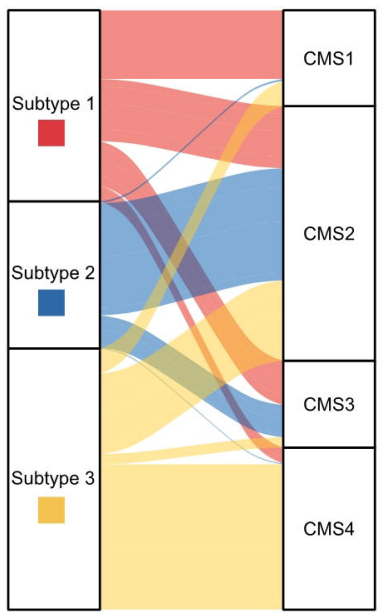




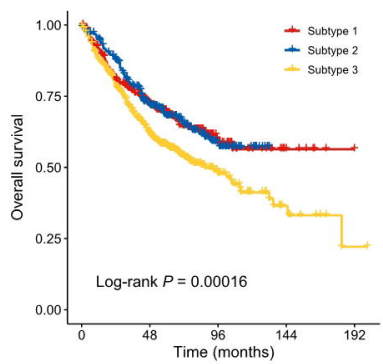

Number at risk

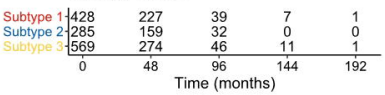

B

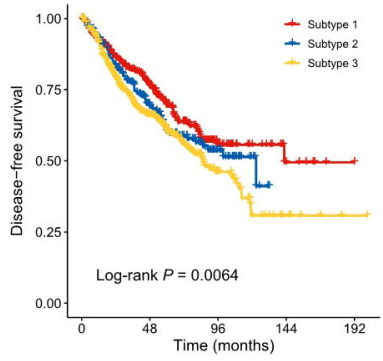

Number at risk

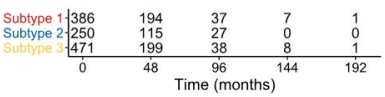

C

Univariate and multivariate Cox regression analysis for DFS

\begin{tabular}{|c|c|c|c|c|}
\hline \multirow{2}{*}{ Factors } & Univariate analysis & \multicolumn{3}{|c|}{ Multivariate analysis } \\
\hline & $\mathrm{HR}(95 \% \mathrm{CI})$ & $P$ & $\mathrm{HR}(95 \% \mathrm{Cl})$ & $P$ \\
\hline \multicolumn{5}{|l|}{ Age (years) } \\
\hline$\leq 60$ & 1 (reference) & & & \\
\hline$>60$ & $1.106(0.881-1.388)$ & 0.386 & & \\
\hline \multicolumn{5}{|l|}{ Gender } \\
\hline Male & 1 (reference) & & & \\
\hline Female & $0.887(0.724-1.087)$ & 0.246 & & \\
\hline \multicolumn{5}{|l|}{ Primary site } \\
\hline Right colon & 1 (reference) & & & \\
\hline Left colon & $1.089(0.799-1.484)$ & 0.591 & & \\
\hline Rectum & 1 & 1 & & \\
\hline \multicolumn{5}{|l|}{ TNM Stage } \\
\hline 1 & 1 (reference) & & 1 (reference) & \\
\hline "I & $1.027(0.734-1.440)$ & 0.874 & $3.957(0.962-16.270)$ & 0.057 \\
\hline III & $1.501(1.071-2.104)$ & 0.019 & $7.286(1.786-29.718)$ & 0.006 \\
\hline \multicolumn{5}{|l|}{ MMR status } \\
\hline PMMR & 1 (reference) & & 1 (reference) & \\
\hline dMMR & $0.514(0.290-0.910)$ & 0.022 & $0.675(0.374-1.218)$ & 0.192 \\
\hline \multicolumn{5}{|l|}{ TMEC subtype } \\
\hline Subtype 1 & 1 (reference) & & & \\
\hline Subtype 2 & $1.220(0.923-1.613)$ & 0.163 & $1.769(1.096-2.855)$ & 0.020 \\
\hline Subtype 3 & $1.456(1.153-1.839)$ & 0.002 & $1.722(1.121-2.646)$ & 0.013 \\
\hline
\end{tabular}

Type $2 \mathrm{~T}$ helper cell

Type $17 \mathrm{~T}$ helper cell

Type $1 \mathrm{~T}$ helper cell

$T$ follicular helper cell Resting natural killer cell

Resting dendritic cell

Regulatory T cell

Plasmacytoid dendritic cell Neutrophil

Natural killer T cell Monocyte

Memory B cell MDSC Mast cell

M2 macrophage M1 macrophage M0 macrophage Immature B cell Gamma delta T cell Fibroblast Eosinophil -

Endothelial cell Effector memeory CD8 T cell Effector memeory CD4 T cell Central memory CD8 T cell Central memory CD4 T cell Activated natural killer cell Activated dendritic cell Activated CD8 T cell Activated CD4 T cell Activated $B$ cell

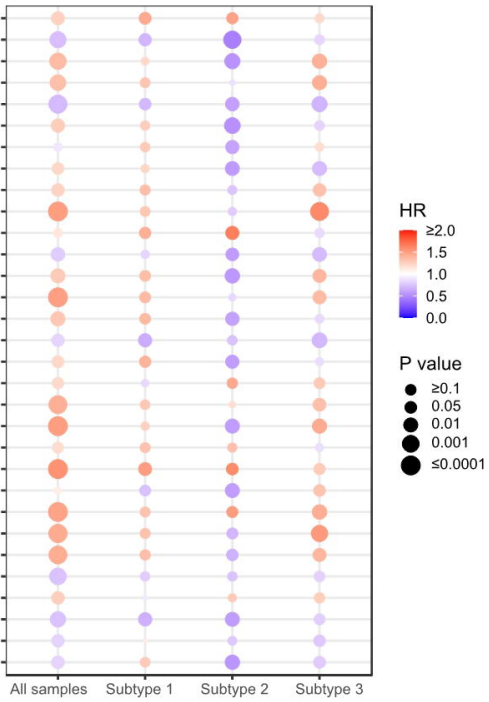

E

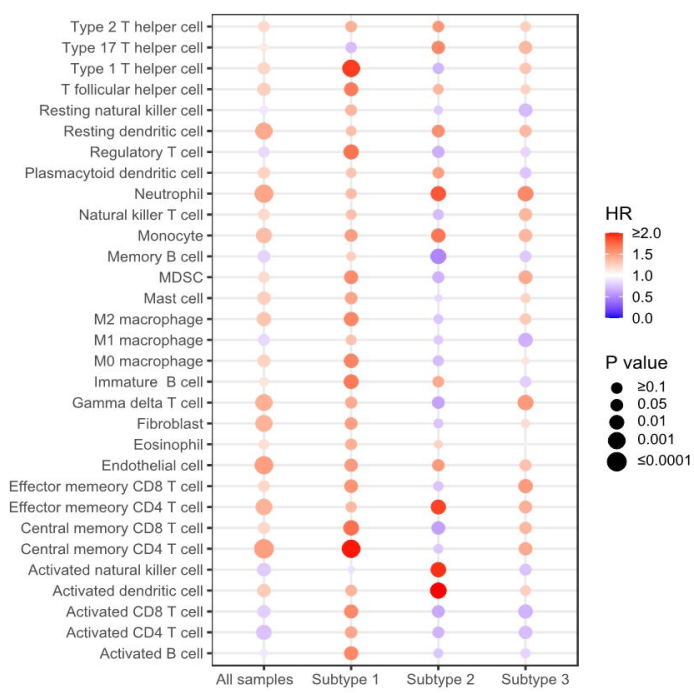


A Mutation load B Neoantigen load C scnVload
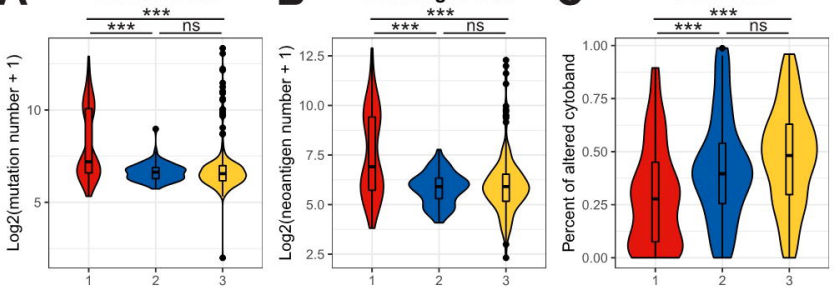

D

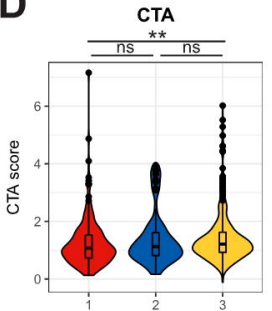

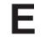

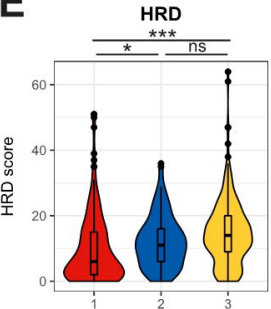

$\mathbf{F}$

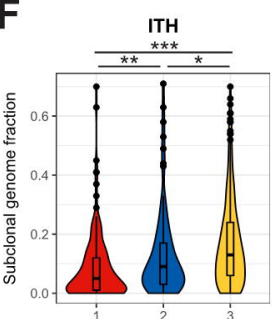

G

Tumor necrosis

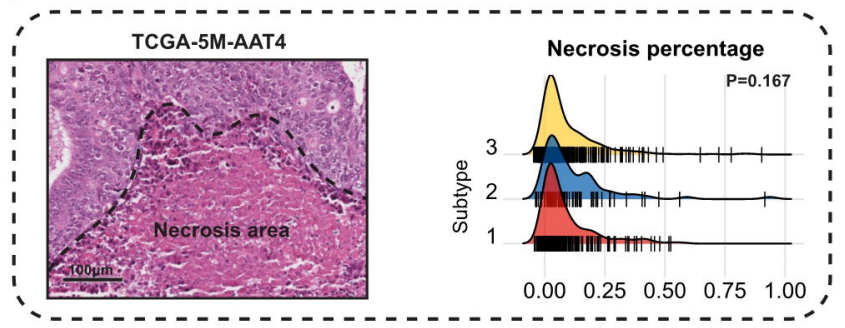

$H$

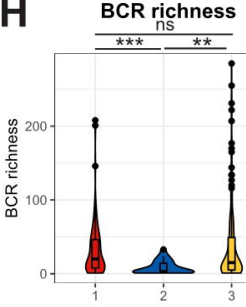

TCR richness

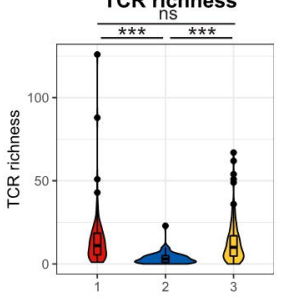

Spearman correlation
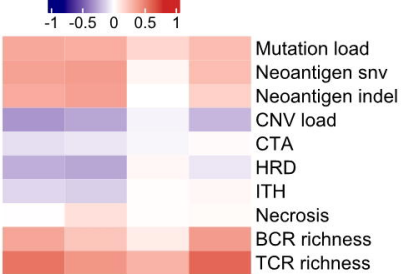

TIL

Stromal cells

PDCD1

LAG3

CTLA4

CD274

TIGIT

PDCD1LG2

CD276

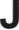
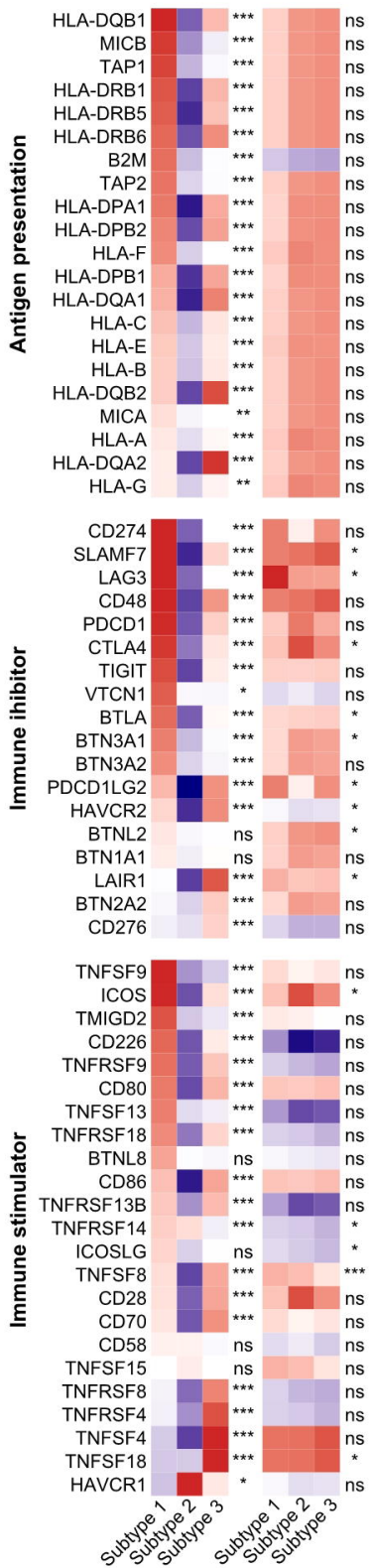

Expression value Log2 (copy number)

$\begin{array}{llll}-2 & -1 & 0 & 1\end{array}$

$\begin{array}{llll}-1 & -0.5 & 0 & 0.5\end{array}$ 
A

Subtype 1 vs. Subtype 2

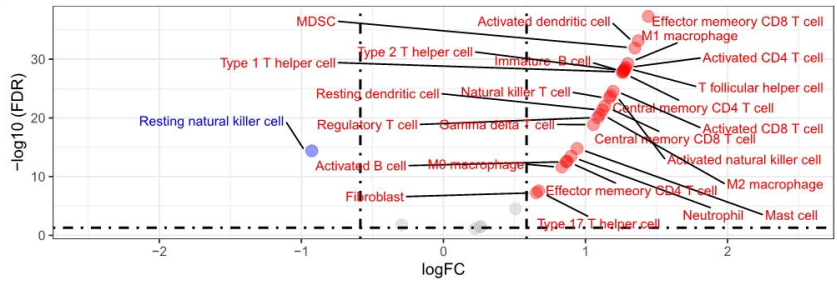

Subtype 1 vs. Subtype 3

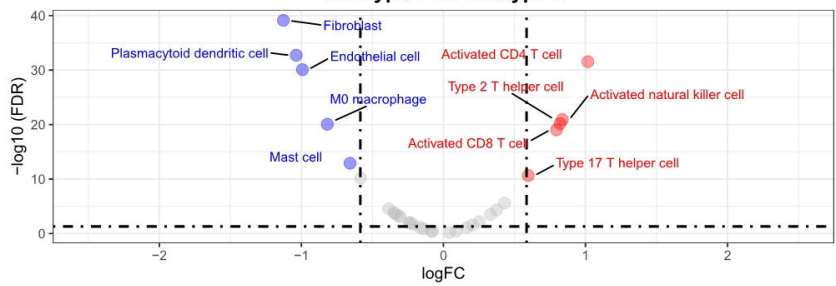

Subtype 2 vs. Subtype 3

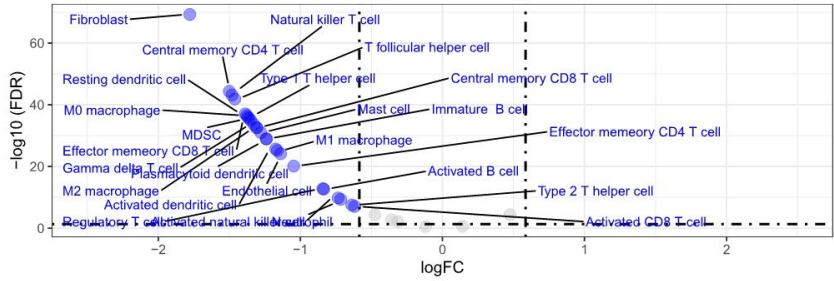

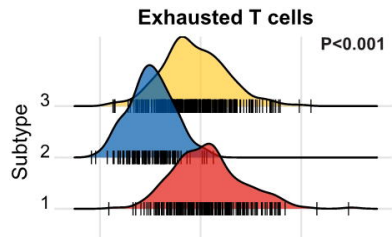

$\begin{array}{lll}0.0 & 0.4 & 0.8\end{array}$

Exhausted T cells/CD8 T cells

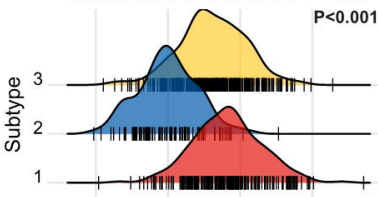

$\begin{array}{lllll}0.0 & 0.1 & 0.2 & 0.3 & 0.4\end{array}$
C

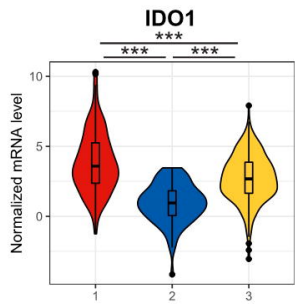

D

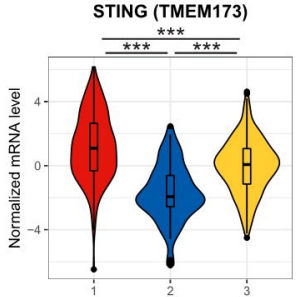

E

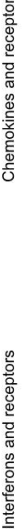

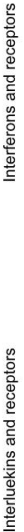

IL12R

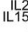

227RA

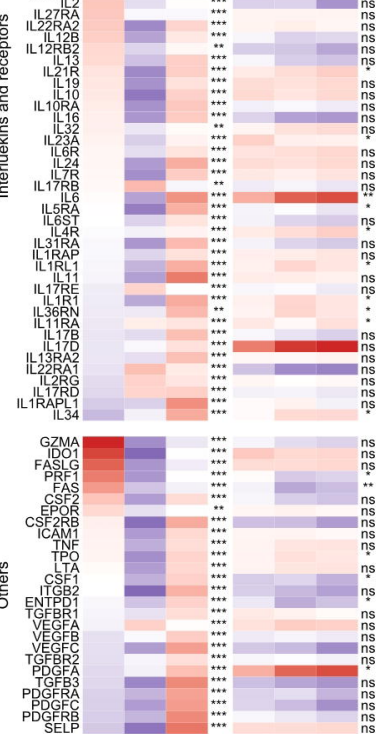

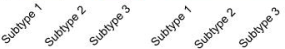

Expression value Log2 (copy number) 
$\begin{array}{lllll}\text { A } & \text { Subtype 1 } & \text { Subtype 2 } & \text { Subtype 3 } & \text { B }\end{array}$

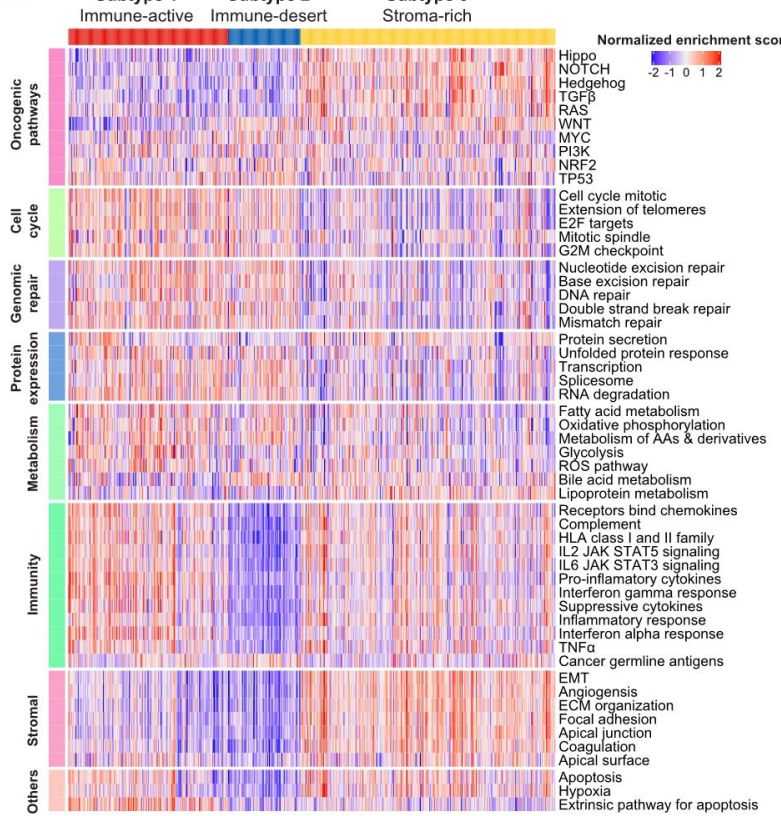

MSS/MSI-L samples

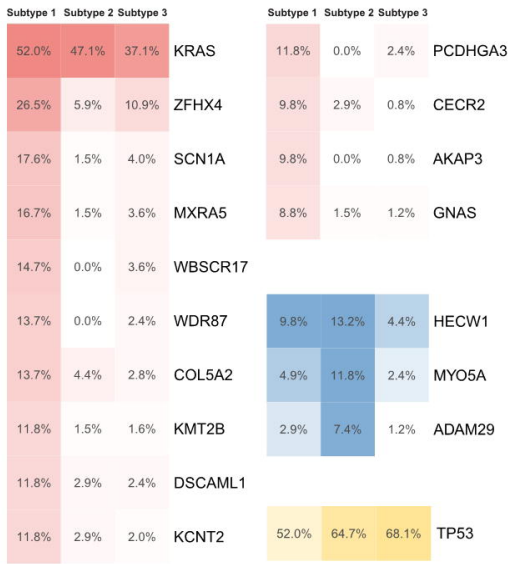

C
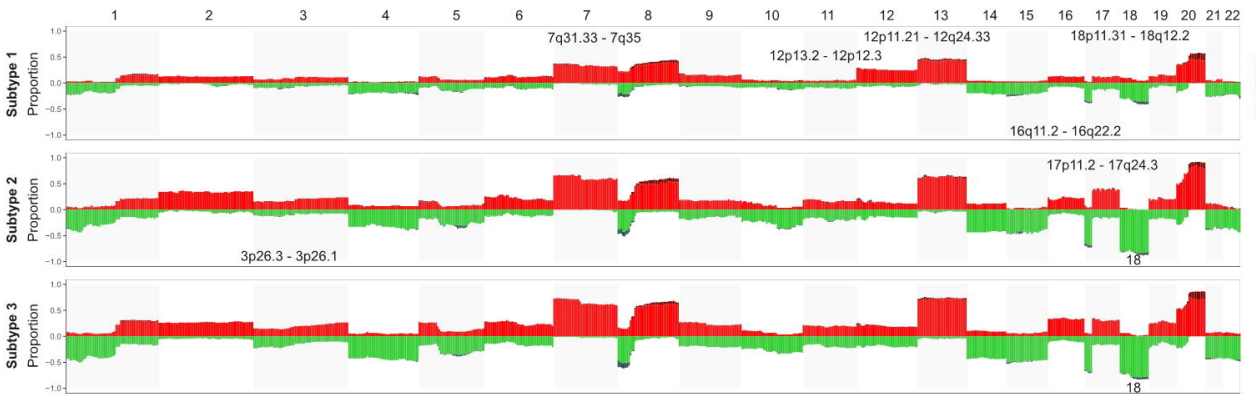

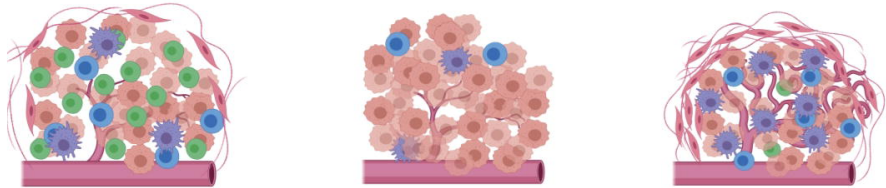

Subtype 1

Immune-active

Proportion

Clinical features
Subtype 2

Immune-desert

$20 \%$

More left colon, more CMS2
Subtype 3

Stroma-ric

$47 \%$

Advanced stages, poor prognosis, more CMS4

\section{Infiltration of immune-suppressive cells}

Lack of immune-promotive cells

Increase of immune-suppressive cells

Older, more right colon, more dMMR/MSI-H, more CMS1

\section{Immune suppressive mediators secretion}

High immune-suppressive cytokine level Low immune-promotive cytokine level

\section{Defective antigen presentation}

Defected tumor immunogenicity

Down-regulation of antigen presentation pathway

Tolerance and immune deviation

Upregulating immunoinhibitors

Downregulating immunostimulators

Specific genomic alteration

Mutations

Somatic copy-number gains/amplifications

Somatic copy-number loss/deletions
KRAS/ZFHX4/KMT2B/MXRA5/DSCAML1/S CN1A/HECW1/WDR87/WBSCR17/PCDHG A3/GNAS/AKAP3

Chr12p13.33-12q24.33/Chr18p11.32$18 q 21.1$
MYO5A/COL5A2/KCNT2/CECR2/ADA

M29

Chr7q31.33-7q35/Chr17q21.32-

$17 q 24.3$

Chr18
$+$

$+$

-

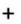

$-$

Chr7q31.33-7q35

Chr18 